\title{
SATISFAÇÃO DO USO DE APARELHO DE AMPLIFICAÇÃO SONORA INDIVIDUAL NO RECONHECIMENTO DE FALA EM PACIENTES PROTETIZADOS BILATERALMENTE
}

\section{SATISFACTION OF THE USE OF INDIVIDUAL SOUND AMPLIFICATION EQUIPMENT IN THE SPEECH RECOGNITION IN BILATERALLY PROTEASE PATIENTS}

\author{
Paloma Gonçalves Santos ${ }^{\mathrm{i}}$; Rachel Costa Façanha ${ }^{1}$
}

RESUMO: O aparelho de amplificação sonora individual na perda auditiva neurossenssorial bilateral tem como função amplificar o som e melhorar o reconhecimento e percepção da fala, evitando isolamento social, e trazendo melhora na qualidade de vida. Teve como objetivo verificar a satisfação do usuário de aparelho de amplificação sonora individual bilateral no desempenho das habilidades auditivas para a compreensão da fala. Foram coletados dados de 30 prontuários de clientes com perda auditiva neurossenssorial bilateral, selecionados aleatoriamente, com histórico de acompanhamento de adaptação de prótese auditiva na empresa em que foi feita a verificação e aquisição. Para avaliar a satisfação do usuário, foi utilizado o questionário Satisfaction With Amplification in Daily Life (SADL) intrumento utilizado na pesquisa e pela na empresa para o registro e arquivo dos dados de acompanhamento. Os resultados obtidos evidenciaram que os clientes encontram-se satisfeitos em todas as subescalas do SADL, sendo que a subescala Serviço e Custo $98,86 \%$ e Efeitos Positivos $96 \%$ para a compreensão da fala obtiveram maior média de satisfação das respostas dos usuários. Para todos os itens de análise de satisfação, foi realizado o cálculo estatístico apresentando que a maioria dos sujeitos demonstraram estar satisfeitos com seus aparelhos auditivos. Constatou-se através dos prontuários verificados, que os clientes estão satisfeitos e consequentemente sendo beneficiados com o uso dos aparelhos auditivos, implicando um bom resultado de adaptação para a melhora da qualidade de vida e na compreensão da fala.

PALAVRAS-CHAVES: Perda Auditiva Neurossenssorial; Percepção Auditiva; Aparelho de Amplificação Sonora Individual.

\footnotetext{
${ }^{1}$ Fonoaudióloga, egressa do curso de Fonoaudiologia da Universidade Ceuma. E-mail: santoospaloma@gmail.com

${ }^{2}$ Fonoaudióloga, Mestre em saúde coletiva pela Universidade Federal do Maranhão (UFMA), Docente do curso de Fonoaudiologia da Universidade Ceuma. E-mail: rachel.facanha@ceuma.br
} 
ABSTRACT: The apparatus of individual sound amplification in bilateral sensorineural hearing loss has the function of amplifying sound and improving speech recognition and perception, avoiding social isolation, and improving the quality of life. Had as objective to verify the satisfaction of the user of bilateral individual sound amplification equipment in the performance of auditoryabilities for speech comprehension. This was a descriptive, transverse and quantitative study. Data were collected from 30 patients' records with bilateral neurosensory hearing loss, randomly selected, with a history of hearing aid adaptation in the company where the verificatio ${ }^{\mathrm{ii}} \mathrm{n}$ and acquisition was performed. To evaluate user satisfaction, the Satisfaction With Amplification in Daily Life questionnaire was used to record customer follow-up data with more than one month of use of individual sound amplification equipment. The results showed that clients were satisfied in all subscales of SADL, and the subscale Service and Cost $98.86 \%$ and Positive Effects $96 \%$ for speech comprehension obtained a higher average satisfaction of the users' responses. For all items of satisfaction analysis, a statistical calculation was performed showing that the majority of the subjects demonstrated to be "satisfied" with their hearing aids. It was verified through verified records that clients are satisfied and consequently benefited from the use of hearing aids, implying a good adaptation result to improve quality of life and speech comprehension.

KEYWORDS: Sensorineural Hearing Loss; Auditory Perception; Individual Sound Amplifier. 


\section{Introdução}

O termo neurossensorial é utilizado hoje para denominar perda de percepção acústica que envolve as lesões sensoriais e neurais. O aparelho de transmissão de som encontra-se normal, no entanto, há uma alteração na qualidade do som e tem uma melhor conservação da audição para sons graves, e audição mais proeminente em sons agudo. Sendo assim a discriminação auditiva geralmente está comprometida de forma variável (LOPES FILHO et al, 2013).

O reconhecimento da fala depende da integridade do sistema auditivo periférico e central e da combinação de pistas como a intensidade, faixa de frequência dos fonemas, a prosódia, a familiaridade o tempo do sinal acústico, a com o vocabulário e o contexto linguístico (MAZZOCHI; AITA, 2013).

Indivíduos com perda auditiva neurossensorial geralmente é necessário o tratamento baseado em habilidades de autocuidado, como, por exemplo, o uso eficaz do aparelho de amplificação sonora individual (AASI) (FERRARI et al, 2015).

A técnica de seleção e adaptação do AASI só será eficaz e terá bons resultados se o paciente fizer uso concreto deste dispositivo. Para isto, é imprescindível que o mesmo esteja tendo vantagem e esteja satisfeito com os resultados sentidos (AURÉLIO et al, 2012).

O método de reabilitação auditiva observa, entre outros fatores, a adaptação de Aparelhos de Amplificação Sonora Individual (AASI). Estes dispositivos têm sido feitos e aprimorados a cada dia com o objetivo de diminuir os efeitos danosos da perda auditiva (ALMEIDA; IORIO, 2003).

O desempenho com a prótese auditiva relatada pelo usuário informa um encaminhamento aos profissionais quanto às medidas necessárias a serem tomadas, proporcionando o reconhecimento das vantagens oferecidas por estes dispositivos em relação às dificuldades auditivas, evitando o abandono e, consequentemente, garantindo a satisfação do paciente (AURÉLIO et al, 2012).

Diante do exposto, o objetivo deste estudo foi verificar a satisfação e benefício do usuário de aparelho de amplificação sonora individual(AASI) bilateral no desenvolvimento das habilidades auditivas para a compreensão da fala através do instrumento de avaliação do nível de satisfação de usuários de AASI, denominado SADL, versão em Português Brasileiro para administrar em prontuários de uma clinica privada de aparelhos auditivos em São Luís. 


\section{Material e métodos}

A pesquisa foi cadastrada na Plataforma Brasil, analisada e aprovada pelo Comitê de Ética em Pesquisa (CEP) do Hospital Universitário da Universidade Federal do Maranhão, HU-UFMA sob o processo $\mathrm{n}^{\circ} 2.155 .612$, de 26 de junho de 2017, com parecer consubstanciado $\mathrm{n}^{\circ}$ 62898716.8 .0000 .5086 .

A pesquisa foi do tipo descritiva transversal e quantitativa.

O estudo foi realizado com a pesquisa de 30 prontuários de um centro auditivo, empresa especializada na comercialização, seleção e adaptação de aparelhos de amplificação sonora individual, que realiza acompanhamento de usuários de aparelhos de amplificação sonora individual (AASI), registrados em um questionário próprio da empresa e arquivados no computador. Nestes, encontramos dados sobre satisfação de uso, manutenção e ajustes, favorecendo o benefício para a melhora da comunicação e da qualidade de vida.

Os prontuários foram consultados sob autorização do proprietário da empresa, com a garantia do sigilo, sendo dispensado o termo de consentimento livre e esclarecido. Foram selecionados aqueles que atenderam aos seguintes critérios de inclusão: registros de satisfação quanto ao uso de AASIs bilaterais de pacientes que apresentaram diagnóstico de Perda Auditiva Sensorioneural Coclear Bilateral, independente do gênero, de grau moderado, com índice de porcentagem de fala entre 60 e $88 \%$.

Como instrumento de coleta de dados, foi utilizado o questionário Satisfaction With Amplification in Daily Life (SADL), validado por Alexander (2001), na versão em português, para administrar em usuários de AASI. Foi elaborado com a intenção de quantificar a satisfação global com o aparelho de amplificação sonora individual e de gerar um perfil de satisfação. A vantagem do $S A D L$ é que se trata de um questionário viável para uso clínico, pois é relativamente curto, contendo apenas 15 questões, que acessam a natureza multidimensional da satisfação, permitindo a identificação de áreas de problemas (CASTIQUINI et al,2011).

Os resultados foram categorizados conforme as respostas: (A) para nada, (B) para um pouco, (C) para de alguma forma, (D) para mediante, (E) para considerável, (F) para muito e $(\mathrm{G})$ para muitíssimo. Como resultado da intervenção com o AASI, satisfação é um conceito complexo que não reflete somente a percepção do paciente com relação ao beneficio do próprio dispositivo (inclui o desempenho, conforto, aparência, etc), mas também pode retratar a questões externas ao tratamento, como a qualidade do serviço, o ambiente de atenção, o custo, o valor percebido, e a 
Revista Ceuma Perspectivas, Edição Especial. V Congresso de Saúde e Bem Estar Ceuma. Vol. 30, n02, 2017. ISSN Eletrônico: 2525-5576.

popularidade do fornecedor (CASTIQUINI et al,2011). O questionário apresenta 15 itens divididos em quatro subescalas, descritas a seguir:

- Efeitos positivos (questões 1, 3, 5, 6, 9 e 10): seis itens referentes ao benefício acústico e psicológico;

- Serviço e custo (questões 12, 14 e 15): três itens referentes à competência profissional, preço do produto e número de consertos;

- Fatores negativos (questões 2, 7 e 11): três itens referentes à amplificação de ruído ambiental, presença de microfonia e uso do telefone;

- Imagem pessoal (questões 4, 8 e 13): três itens referentes à estética e ao estigma do uso do AASI.

Todos os usuários realizaram inicialmente exames auditivos para a verificação de tipo, grau e porcentagem de reconhecimento de fala (IPRF), com o uso do equipamento audiômetro AD 229 e, com última calibração em julho de 2017, registrados em prontuários pela fonoaudióloga responsável pelo local. Posteriormente foram selecionados e adaptados aparelhos auditivos da marca ARGOSY e registrados os acompanhamentos para a satisfação quanto ao uso dos AASIs e a melhora da qualidade de comunicação e de vida. A maioria dos prontuários analisados, que atenderam aos critérios de inclusão da pesquisa, foi de idosos, percebendo-se registro de respostas prevalentes para as seguintes categorias: muitíssimo satisfeito $(\mathrm{G})$, para nada satisfeito (A) e para consideravelmente satisfeito (E).

Com isto, os resultados da análise dos dados obtidos dos 30 prontuários selecionados foram demonstrados respeitando estas categorias. Para efetuar a análise referente às 15 questões de satisfação, foi utilizada uma escala de 7 pontos de igual intervalo, correspondendo em "nada" a "muitíssimo". Para 11 questões o "muitíssimo" indicou total satisfação, sendo este colocado com o valor 7, enquanto "nada" indiciou total insatisfação e foi pontuada com o valor 1 . As outras quatro questões $(2,4,7$ e 13) foram invertidas, nas quais "muitíssimo" indicou total insatisfação, sendo colocado como valor 1 e, "nada" indicou total satisfação, sendo colocada com valor 7.

A análise dos dados foi desenvolvida através de dados tabulados no Microsoft Excel 2013 e a análise estatística dos resultados foi realizada por meio do programa estatístico Sphynx (versão 5). Foram calculadas as médias, medianas máxima e mínima, desvios padrões das subescalas. Para verificar a relação de satisfação dos usuários de prótese auditivas com as subescalas, foi realizado o teste Qui-quadrado. Todas as associações e comparações foram consideradas como estatisticamente significativas quando alpha foi inferior a $5 \%(<0,005)$. 
Revista Ceuma Perspectivas, Edição Especial. V Congresso de Saúde e Bem Estar Ceuma. Vol. 30, n02, 2017. ISSN Eletrônico: 2525-5576.

\section{Resultados e discussão}

Foram inclusos na pesquisa 30 prontuários de pacientes adaptados bilateralmente com AASIs, com média de idade de 73 anos, média de mais um mês de tempo de uso dos AASIs. A Tabela 1 descreve os resultados obtidos pelos sujeitos para a pontuação global e para as quatro subescalas do SADL: média, desvio-padrão, mediana de valores máximos e mínimos.

\begin{tabular}{|c|c|c|c|c|c|}
\hline$\overline{\text { SADL }}$ & $\mathrm{N}$ & Média & DP & Mínimo & Máximo \\
\hline Global & 30 & 5.6 & 0.7 & 4.5 & 7,0 \\
\hline $\begin{array}{l}\text { Efeitos } \\
\text { positivos }\end{array}$ & 30 & 6.7 & 0.71 & 6.0 & 7,0 \\
\hline $\begin{array}{l}\text { Serviço e } \\
\text { custo }\end{array}$ & 30 & 6.9 & 0.12 & 6,3 & 7,0 \\
\hline $\begin{array}{l}\text { Fatores } \\
\text { negatives }\end{array}$ & 30 & 3.71 & 0.72 & 3.0 & 7,0 \\
\hline $\begin{array}{l}\text { Imagem } \\
\text { pessoal }\end{array}$ & 30 & 5.26 & 1.26 & 3.0 & 7,0 \\
\hline
\end{tabular}

Fonte: SANTOS, novembro, 2017

Os resultados com relação às subescalas do questionário para a média revelaram maior satisfação para Serviços e Custos (6,9), seguida do Efeito Positivo $(6,7)$ e, logo após, Imagem Pessoal (5.26). Foi observada menor satisfação para a subescala Fatores Negativos (3.71), pontuando uma média de 5.6 para a escala global.

Os percentuais para as subescalas do questionário revelaram maior satisfação para Serviço e Custo $(98,86 \%)$, seguida de Efeitos Positivos $(96 \%)$, Imagem Pessoal $(88,86)$ e Fatores Negativos $(66,66 \%)$, o que corrobora com a Pontuação Global (89\%). Esses resultados do questionário indicam que os usuários estavam muito satisfeitos com o uso do AASI (Gráfico 1).

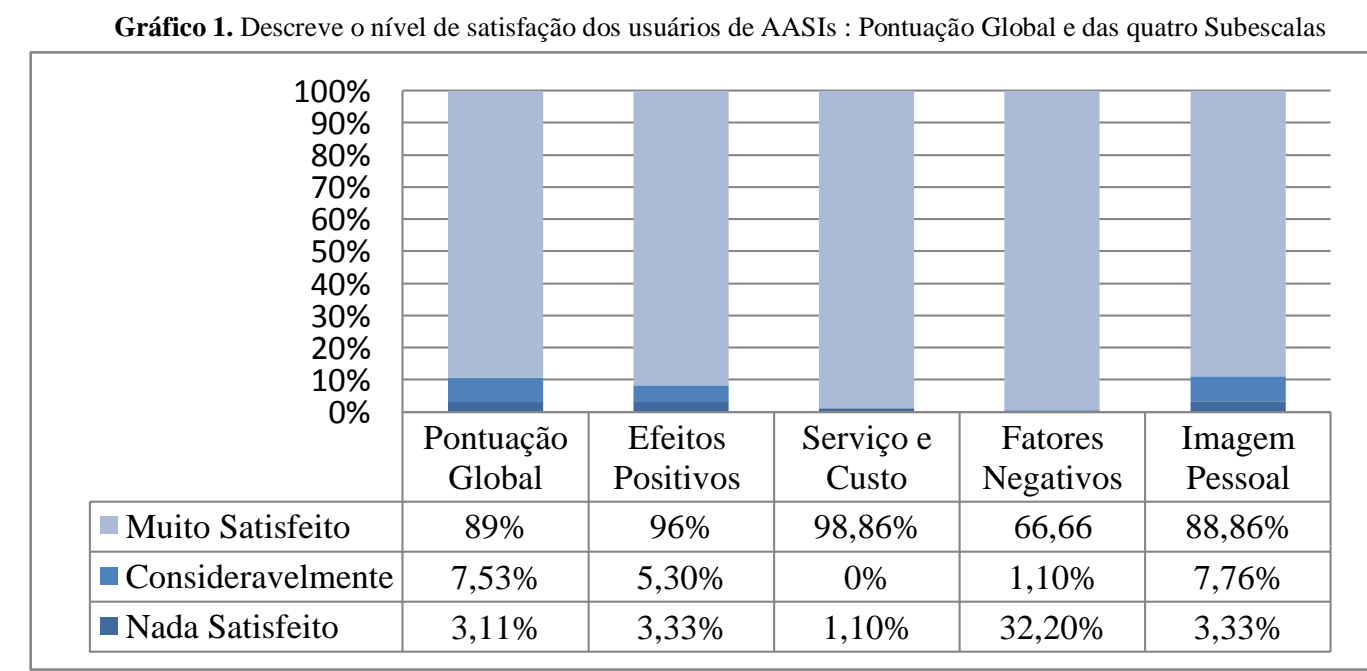

Fonte: SANTOS, novembro, 2017 
Revista Ceuma Perspectivas, Edição Especial. V Congresso de Saúde e Bem Estar Ceuma. Vol. 30, n02, 2017. ISSN Eletrônico: 2525-5576.

Para os dados de satisfação referentes a compreensão de fala, objeto geral deste estudo, 30 $(100 \%)$ referiram estar "muitíssimo satisfeitos" com o benefício de melhora da inteligibilidade de fala com e sem AASI; para entender ao telefone e para a relação custo e benefícios das próteses auditivas. Informaram satisfação relacionada a redução de solicitação de repetições para entender o que falado e a qualidade de som do AASI 29 (96,7\%). Com relação a compensação do uso do dispositivo para a compreensão, 25 (83,3\%), referiram estar "muitíssimo satisfeitos". Poucos usuários, 7 (23,3\%) informaram frustação quanto a captação do ruído para a compreensão da fala (Tabela 2).

Tabela 2: Variáveis referentes à melhora de compreensão de fala com o uso do AASI.

\begin{tabular}{|c|c|c|c|}
\hline Variáveis de Compreensão de Fala & $\mathbf{f}$ & $\%$ & p-valor \\
\hline Melhora da compreensão de fala com e sem AASI & 30 & $100 \%$ & $<0,01$ \\
\hline Redução de solicitação de repetição de fala para entender & 29 & $96,7 \%$ & $<0,01$ \\
\hline Compensação do aparelho para a compreensão da fala & 25 & $83,3 \%$ & $<0,01$ \\
\hline Satisfação com a qualidade do som para compreensão da fala & 29 & $96,7 \%$ & $<0,01$ \\
\hline Frustação quanto a captação do ruído para a compreensão da fala & 7 & $23,3 \%$ & $<0,01$ \\
\hline Melhora da compreensão de fala ao telefone & 30 & $100 \%$ & $<0,01$ \\
\hline Relação custo e benefícios para a compreensão da fala & 30 & $100 \%$ & $<0,01$ \\
\hline
\end{tabular}

Fonte: SANTOS, novembro, 2017

Para a correlação entre a satisfação e a melhora conhecimento de compreensão de fala o pvalor foi $<0,01$ para todos os itens possivelmente por ser uma amostra pequena.

Nesta pesquisa utilizou-se o questionário Satisfaction with Amplification in Daily Life $(S A D L)$ para a obtenção da análise dos resultados. Este é sugerido em artigos que falam sobre a praticidade, facilidade e eficácia desta ferramenta o que corrobora para a veracidade do estudo realizado. Sendo assim, para a validação ou avaliação dos benefícios of erecidos pelo AASI podem ser utilizados questionários de autoavaliação durante o processo de adaptação, podendo empregá-los em diversas situações como na rotina clínica, na triagem auditiva, entrevista inicial, aconselhamento e na avaliação da irrefutabilidade dos programas de reabilitação audiológica (BASÍLIO,2015).

Contraditório a esta pesquisa e outros estudos, para a boa aplicabilidade do questionário, um estudo (BEATON et al, 2002) sugeriu que sejam feitas mais pesquisas com uma quantidade pequena de sujeitos pertencentes ao público alvo (SILVA, D.; SILVA, V.; AURÉLIO, 2013) para garantir a boa reprodução do questionário.

O questionário SADL foi elaborado com o intuito de quantificar a satisfação global com o AASI e de gerar um perfil de satisfação em cada um dos quatro maiores domínios da satisfação 
(CASTIQUINI et al, 2011). Dessas quatro áreas de domínios de satisfação, nesta pesquisa foi obtido um resultado satisfatório para as subdivisões das escalas efeitos positivos, serviço e custo, efeitos negativos e imagem pessoal.

Os resultados com relação às subescalas do questionário revelaram maior satisfação para Serviço e Custo, seguida de Efeitos Positivos, Imagem Pessoal e Fatores Negativos. Os achados da subescala que avalia a Imagem Pessoal teve média maior à encontrada em outro estudo equivalente já realizado (SILVA, D.; SILVA, V.; AURÉLIO, 2013). Outros estudos (VEIGA; MERLO; MENGUE, 2005; COX; ALEXANDER, 1999; CARVALHO, 2007; SOARES et al, 2007) indicam a satisfação para este grupo de questões que abordam o estigma da prótese auditiva e estes resultados estão de acordo com o tipo de serviço oferecido aos usuários, pois assim como este, são resultados de avaliação de uma empresa particular em que os pacientes são orientados da melhor forma a adquirir um aparelho ideal para sua perda auditiva, mas que também agrade esteticamente o usuário.

Os resultados destacam que é muito importante acompanhar a satisfação do usuário de AASI para as técnicas clínicas, pois ao reconhecer fatores que contribuem para a satisfação e ao tentar favorecer tais atributos aos procedimentos envolvidos, tem-se o potencial de obter um resultado mais eficaz nos serviços de saúde, além de uma melhora na adaptação do sujeito frente ao produto que está utilizando.

Mesmo em estudos (CASTIQUINI et al, 2011; VEIGA; MERLO; MENGUE, 2005) com a concessão de AASI gratuitamente, pelo SUS ou o exército brasileiro, os resultados demonstraram que a escala de estigma do aparelho apresentaram-se "muito satisfeitos".

No que concerne à subescala serviços e custos, teve média maior do que as encontradas em outros estudos (LESSA et al, 2010; CASTIQUINI et al, 2011), assim como a de Efeitos Positivos (VEIGA; MERLO; MENGUE, 2005; CARVALHO, 2007; SOARES et al, 2007). Para Fatores Negativos, outras pesquisas (VEIGA; MERLO; MENGUE, 2005; SOARES et al, 2007; LESSA et al, 2010) encontraram médias superiores a do presente estudo. Esta última se deve ao fato de apresentar um menor score por se avaliar aspectos considerados problemáticos na adaptação.

Os resultados dos prontuários avaliados na presente pesquisa estão prevalentes para "muito satisfeitos" em comparação aos resultados da pesquisa original do SADL (COX; ALEXANDER, 2011). De acordo com outras pesquisas (MONDELLI; MAGALHÃES; LAURIS, 2011), os resultados obtidos foram equivalentes a esta, corroborando com os scores de 1 a 7 . 
A compreensão dos sons da fala engloba a recepção e interpretação dos padrões de fala; a distinção entre os sons de diferentes espectros, durações, peculiaridades temporais, formas sequenciais e ritmo; o reconhecimento, a memorização e a compreensão de unidades de fala dentro de determinado sistema linguístico (MAGALHÃES; CIMONARI; NOVAES, 2007).

Outro estudo corrobora com estas informações, destacando que o reconhecimento de fala não está envolvido somente com sucesso na adaptação de AASIs, mas sim que envolve múltiplos aspectos. Refere, ainda, que não podemos limitar somente à melhora na compreensão, mas também aos aspectos relacionados a todo o processo de comunicação do usuário de prótese auditiva, propiciando um nível de satisfação quanto a sua nova condição de ouvinte (PRATES; IÓRIO, 2006). Uma pesquisa realizada com o propósito de avaliar o reconhecimento de fala, benefícios e satisfação de usuários de AASI, apresentou resultados para a discriminação de fala equivalentes aos obtidos no presente estudo, estando relacionados a melhora significativa da percepção da fala. Isso foi comprovado com os resultados relacionados ao benefício do usuário de prótese auditiva e a compreensão, que apresentaram-se estatisticamente significantes para o grau de satisfação "muitíssimo", com um percentual baixo de satisfação para o Efeito Negativo referente a frustração quando escuta sons que não gostariam de ouvir (COSTA et al, 2007).

A alta satisfação dos usuários em relação a discriminação de fala foi demonstrada nos registros dos prontuários analisados, sinalizando os benefícios que uma prótese auditiva bem adaptada pode favorecer à melhora da qualidade de vida e de inserção social. Em estudo realizado por Costa (2007) corrobora com o resultado dos benefícios analisados por esta pesquisa, o que menciona que o AASI é extremamente fundamental para a melhora da comunicação, melhorando a sociabilização de deficientes auditivos, desde que bem adaptado.

Apesar deste estudo ter resultados confiáveis com a utilização do SADL versão em Português Brasileiro como instrumento para avaliar o nível de satisfação e os benefícios de usuários de AASIs para a compreensão, novas pesquisas são necessárias para complementar estes achados, englobando um maior número de dados registrados e também outros grupos de usuários de AASI.

\section{Conclusões}

Após a análise dos resultados obtidos, pôde-se concluir que os usuários estão tendo benefícios com o uso dos aparelhos auditivos e mostram-se muito satisfeitos com os resultados da adaptação. 
A aplicação de questionários como forma de uma avaliação subjetiva é de extrema importância para a verificação dos benefícios e da satisfação dos usuários de próteses auditivas, pois podemos analisar subjetivamente a correta seleção e adaptação dos aparelhos auditivos, podendo ser incorporada à rotina clínica dos fonoaudiólogos.

Os resultados encontrados demonstram a importância dos registros obtidos pelos prontuários para a avaliação individual das consequências psicossociais geradas pela deficiência auditiva, viabilizando a busca de estratégias para a sua minimização e cooperando para possíveis pesquisas.

Conclui-se ainda, que uma prótese auditiva bem adaptada favorece na melhora significativa da compreensão de fala, minimizando os efeitos que a deficiência auditiva pode provocar na socialização.

\section{Referências}

ALEXANDER, G.C. Validation of the SADL questionnaire, 2001.

ALMEIDA, K.; IORIO, M.C.M. Próteses Auditivas: Fundamentos Teóricos e Aplicações Clínicas. São Paulo: Lovise, 2003.

AURÉLIO, F.S. et al. Satisfaction of patients fit with a hearing aid in a high complexity clinic. Braz J Otorhinolaryngol, 2012.

BAZILIO, F.O. Satisfação do usuário de aparelho auditivo em sua vida diária- união da vitória -pr e região, 2015.

BEATON, D. et al. Recommendations for the Cross-Cultural Adaptation of Health Status Measures. American Academy of Orthopaedic Surgeons and Institute for Work \& Health. Revised March, 2002.

CARVALHO, J.S.A. Satisfação de idosos com aparelhos auditivos concedidos no estado do Tocantins. Arq Int Otorrinolaringol, 11(4):416-26, 2007.

CASTIQUINI, F. et al. Avaliação do nível de satisfação de usuários de aparelhos de amplificação sonora individuais dispensados pelo Sistema Único de Saúde. Rev Socied Brasil de Fonoaudiologia, 16 (2) :152-9, 2011.

COSTA, M.H.P. et al. Avaliação do Benefício da Prótese Auditiva Digital e da Percepção da Desvantagem Auditiva ou "Handicap" em Idosos não Institucionalizados Arq. Int. Otorrinolaringol. / Intl. Arch. Otorhinolaryngol., São Paulo, v.11, n.2, p. 159-168, 2007. 
Revista Ceuma Perspectivas, Edição Especial. V Congresso de Saúde e Bem Estar Ceuma. Vol. 30, n02, 2017. ISSN Eletrônico: 2525-5576.

COX, R.M.; ALEXANDER, G.C. Measuring satisfaction with amplification in daily life: the SADL scale. Ear Hear, 20(4):306-20, 1999.

COX, R.M.; ALEXANDER, G.C. Validation of the SADL questionnaire. Ear Hear. 22(2):151-60, 2001.

FERRARI, D.V. et al. Teste prático das habilidades de manuseio do aparelho de amplificação sonora individual (PHAST): resultados na adaptação e comparação da confiabilidade entre avaliadores, Audiol Commun Res. 20(2):110-5110, 2015.

LESSA, A.H. et al. Satisfação de usuários de próteses auditivas, com perda auditiva de graus severo e profundo. Arq. Int. Otorrinolaringol. / Intl. Arch. Otorhinolaryngol., São Paulo - Brasil, 14, (3): 338-345, 2010.

LOPES FILHO, O.; CAMPIOTO, A.R.; LEVY, C.C.C.; REDONDO, M.C.; ANELLI, W. Tratado de Fonoaudiologia. Editora Manole, São Paulo, (3):3-43, 2013.

MAGALHÃES, L.A.; CIMONARI, P.M.; NOVAES, B.C.A.C. Avaliação de percepção de fala em crianças com deficiência auditiva usuárias de aparelho de amplificação sonora: a questão do instrumento e seus critérios. Rev Soc Bras Fonoaudiol., 12(3):221-32, 2007.

MAZZOCHI, M.T.; AITA, A.D.C. Direcionalidade e reconhecimento de fala no ruído: estudo de quatro casos, Rev. CEFAC. 15(3):689-696, Mai./Jun., 2013.

MONDELLI, M.F.C.G.; MAGALHÃES, F.F.; LAURIS, J.R.P. Adaptação cultural do questionário SADL (Satisfaction with Amplification in Daily Life) para o português brasileiro. Braz J

Otorhinolaryngol., 77 (5):563-72, 2011.

PRATES, L. P. C. S.; IÓRIO, M. C. M. Aclimatização: estudo do reconhecimento de fala em usuários de próteses auditivas. Pró-Fono Revista de Atualização Científica, Barueri (SP), v. 18, n. 3, p.259-266, set./dez., 2006.

SILVA, D.P.C.B.; SILVA, V.B.; AURÉLIO, F.S. Satisfação auditiva de pacientes protetizados pelo Sistema Único de Saúde e benefício fornecido pelos dispositivos. Brazilian Journal of otorhinolaryngology, 79 (5), 2013.

SOARES, D.O. et al. Satisfação dos usuários de prótese auditiva em seu dia-a-dia. Acta Orl, 25(4):290-92, 2007.

VEIGA, L.R.; MERLO, A.R.C.; MENGUE, S.S. Satisfação com a prótese auditiva na vida diária em usuários do sistema de saúde do exército. Rev Bras Otorrinolaringol, 71(1):67-73, 2005. 